\title{
STUDY OF THERMAL STABILITY OF METAL GLASSES OF METAL - METAL AND METAL - METALOID SYSTEMS FOR THERMOMETRY PURPOSES
}

\author{
Bohdan Stadnyk, Dr. Sc., Prof.; Pylyp Skoropad, Dr. Sc., Prof.; \\ Yuriy Nakonechnyi, PhD, Ass.-Prof., Vitaliy Myhaylov, Student \\ Lviv Polytechnic National University, Ukraine
}

\begin{abstract}
The metastable metallic glasses are studied in the paper. Their properties as the material for producing the sensitive elements of thermometric transducers depend on the peculiarities of manufacturing the mentioned material. The selection of modes of glass production and the correction of modes facilitates to increase in the operating temperatures of the transducers or/and extends their service life.
\end{abstract}

Key words: Metal amorphous alloy, Metastable material, Sensitive element, Thermoelectric transducer.

\section{Introduction}

It is known [1] that metal amorphous alloys (metallic glasses) are structurally metastable. This metastability is a function of many technical factors inherent in their manufacture, and several operational factors of using the mentioned metallic glass as a sensitive element of the transducer.

\section{Problem statement and shortcomings}

Therefore, it is quite important to establish criteria for optimizing approaches to:

a) the development of new glasses;

b) the choice of the most optimal from the existing glasses.

This is especially true for the selection of metallic glass suitable for use at temperatures above $300 \mathrm{~K}$, in particular, in the primary temperature transducers of electrical resistance and thermo-EMF.

\section{The purpose of the work}

The aim of the current paper is the study of thermal stability and factors that affect, metastable amorphous alloys to obtain optimal alloy for producing the sensitive element from it.

\section{Investigation of the main characteristics of metal glasses}

Factors influencing the formation and stability of the structure of the amorphous state in metallic glasses can be grouped as follows: thermodynamic, kinetic, and physicochemical. The latter is the least studied, however, the most informative in terms of application in electro thermometry.

\subsection{Investigation of thermal stability}

Analyzing the available references, in particular [2], it can be concluded that the structural stability and properties of metal glasses depend on the kind and state of glass components. Taking to account the well-known empirical dependence of $T_{C} / T_{M}=0.4 \ldots 0.6$, it can be ascertained that the most stable amorphous systems have to be based on the metals with a high melting point. However, this statement is not entirely true. According to the results of the research, the value of the ratio of the average temperature of the transition to the crystalline state of $T_{C r}$ and the melting temperature $T_{M}$ of metallic glasses is not permanent. As it was turned out, the ratio tends to increase for the alloys the composition of which is located in the middle part of the state diagram. According to [2], this is caused by the formation of intermetallic compounds with complex packed crystalline structures.

Since the temperature of the beginning of the transition of metal glasses into the crystalline state $T_{C r}$ is the quite important characteristic, to assess their thermal stability more appropriate is to consider the known empirical dependence connecting $T_{C r}$ with the concentration dependence of enthalpy of vacancy type $\boldsymbol{\Delta} \boldsymbol{H}_{C}[\mathrm{~kJ} /$ mol]: $T_{C r} \approx(7 \ldots 8) \Delta \boldsymbol{H}_{C}$. Based on this dependence analysis, it can be concluded that due to the structural relaxation of the studied materials, their temperature at the beginning of the transition to the crystalline state can change significantly.

Similar to the latter characteristic, the glass transition temperature $T_{g l}$ can serve as a measure of thermal stability of metallic glasses. Thus, for the vast majority of metal amorphous systems, it is true that $T_{g l} /$ $T \geq 0.45$, and it makes it possible to obtain an amorphous structure of materials that are hardened at speeds not exceeding $10^{6} \mathrm{~K} / \mathrm{s}$. Thus, the higher the glass transition temperature and the lower the melting temperature, the more stable would be the amorphous state.

\subsection{Analysis of the model of thermal stability} of the amorphous state

The models of thermal stability of the amorphous state materials are mainly based on the assumption that the rate of transition to the crystalline state, which is determined by diffusion processes, is proportional to the value of the inverse viscosity. Thus, in the theory of entropy viscosity, the dependence of the change in viscosity on temperature is determined as follows: 


$$
\eta=C \exp (\Delta E / S T)
$$

Here $C$ is a constant; $\boldsymbol{\Delta} \boldsymbol{E}$ is the activation energy of viscous character of transition; $\boldsymbol{S}$ is the configuration entropy. In the solid-state at the temperature $T_{C r}$, the entropy practically remains virtually unchanged, and the crystallization of the amorphous alloy mainly occurs at the moment of reaching the critical viscosity, which is equal to $\approx 10^{12} \mathrm{~Pa}+\mathrm{s}$. Therefore, under the condition of increasing the viscosity of the melt, the temperature of glass formation increases, and as a consequence, increases the temperature of transition to the crystalline state of metallic glass.

To determine the value of the temperature of the phase transition $T_{P h}$ of the studied metal glasses, we analyze the temperature dependences of their reduced electrical resistance at a heating rate of $20 \mathrm{~K} / \mathrm{s}$, shown in Fig. 1.
Taking into account the crystallization rate $V_{C r}$ (where $V_{C r}=\Delta\left(R_{T} / R_{273 K}\right) / \Delta T$ ), the phase transition temperature $T_{P h}$ is considered to be the point at which the crystallization rate is maximum. The results of such an analysis for typical metallic glasses are given in the table. 1

\subsection{Methods for studying the kinetics}

of crystallization of metal glasses by their electrical resistance changes

The obtained results of the research indicate that an unambiguous relationship between the alloys' tendency to an amorphous state transition and their resistance to crystallization is absent. It seems that the latter for the studied materials increases with the rate of their hardening.

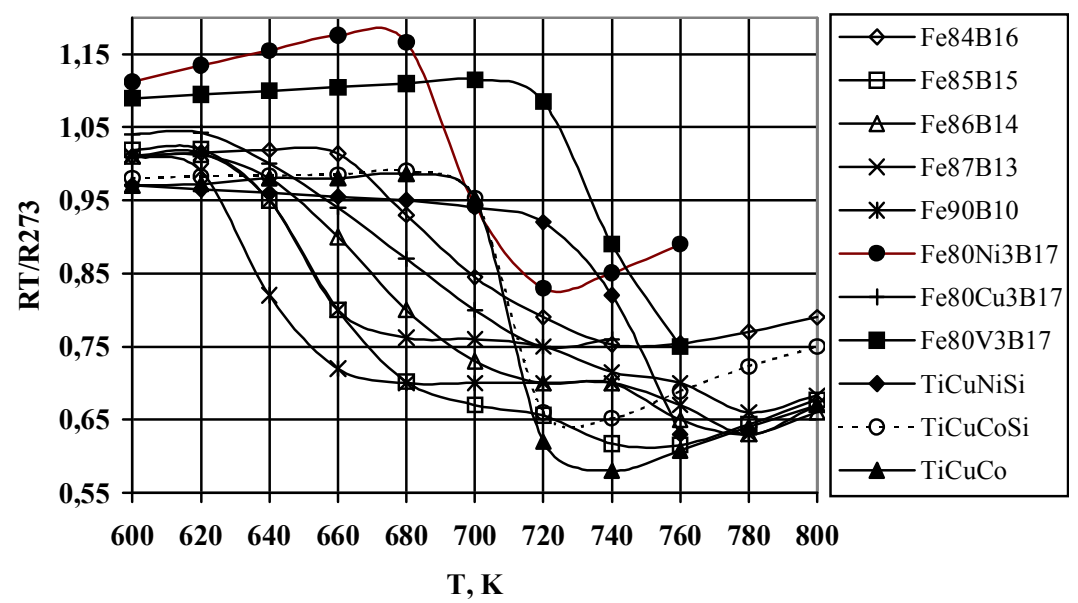

Fig. 1. Typical temperature dependences of the reduced electrical resistance of the studied materials

Table 1

Values of the melting points, glass formation, and phase transition temperatures, and crystallization rate in them for some typical metal glasses of metal-metal and metal-metalloid classes

\begin{tabular}{|c|c|c|c|c|}
\hline Alloys & $T_{M}, \mathrm{~K}$ & $T_{g l}, \mathrm{~K}$ & $T_{P h}, \mathrm{~K}$ & $V_{\mathrm{K}}, \mathrm{K}^{-1}$ \\
\hline $\mathrm{Fe}_{84} \mathrm{~B}_{16}$ & 1888 & 882 & 660 & -0.00223 \\
\hline $\mathrm{Fe}_{85} \mathrm{~B}_{15}$ & 1883 & 879 & $\begin{array}{l}635 \\
750\end{array}$ & $\begin{array}{l}-8.279 \\
-6.064\end{array}$ \\
\hline $\mathrm{Fe}_{86} \mathrm{~B}_{14}$ & 1878 & 877 & $\begin{array}{l}630 \\
775\end{array}$ & $\begin{array}{l}-0.0016 \\
-0.0010\end{array}$ \\
\hline $\mathrm{Fe}_{87} \mathrm{~B}_{13}$ & 1873 & 875 & $\begin{array}{l}620 \\
775 \\
\end{array}$ & $\begin{array}{l}-0.0015 \\
-0.0021 \\
\end{array}$ \\
\hline $\mathrm{Fe}_{90} \mathrm{~B}_{10}$ & 1859 & 868 & $\begin{array}{l}610 \\
780\end{array}$ & $\begin{array}{c}-0.001 \\
-1.63 \cdot 10^{-6}\end{array}$ \\
\hline $\mathrm{Fe}_{80} \mathrm{Ni}_{3} \mathrm{~B}_{17}$ & 1891 & 883 & 680 & -0.0058 \\
\hline $\mathrm{Fe}_{80} \mathrm{Cu}_{3} \mathrm{~B}_{17}$ & 1880 & 878 & $\begin{array}{l}623 \\
717 \\
\end{array}$ & $\begin{array}{l}-0.0022 \\
-0.0035 \\
\end{array}$ \\
\hline $\mathrm{Fe}_{80} \mathrm{~V}_{3} \mathrm{~B}_{17}$ & 1904 & 889 & 720 & -0.0056 \\
\hline Ti-Cu-Ni-Si & 1677 & 883 & 720 & -0.00297 \\
\hline Ti-Cu-Co-Si & 1687 & 788 & 710 & -0.015 \\
\hline $\mathrm{Ti}-\mathrm{Cu}-\mathrm{Co}$ & 1674 & 782 & 700 & -0.0093 \\
\hline
\end{tabular}


To study the kinetics of crystallization of metal amorphous alloys, the method of electrical resistance was applied. For this purpose, a series of isothermal annealing of samples at different temperature values with fixation of the dynamics of changes in their resistance was performed. The process analysis was fulfilled based on the modified Avrami equation [5]:

$$
\begin{aligned}
& \log \left[\ln \left(1-\frac{R A}{R_{A}-R_{K}}\right)^{-1}\right]= \\
& =n\left[\log \left(\tau_{0} \exp \left(\frac{-E_{K}}{k T}\right)\right)^{-1}+\log t,\right.
\end{aligned}
$$

where $\boldsymbol{R}_{\boldsymbol{A}}$ is the initial electrical resistance of the sample in the amorphous state, Ohm; $\boldsymbol{R}(\boldsymbol{t})$ is the electrical resistance of the sample at moment $t, \mathrm{Ohm} ; \boldsymbol{R}_{\boldsymbol{F}}$ is the final electrical resistance of the sample in the crystalline state, Ohm; $\boldsymbol{n}$ is a factor determined by the nature of the nucleation and growth of the crystals; $\tau_{0}$ is the frequency factor, $\mathrm{s} ; \boldsymbol{E}_{\boldsymbol{A} C}$ is the activation energy of the crystallization, $\mathrm{eV} ; \boldsymbol{k}$ is the Boltzmann constant, $\mathrm{eV} / \mathrm{K}$.

To determine the thermal stability of metallic glasses as a function of temperature and operating time, they were subjected to permanent isothermal annealing at a temperature $\boldsymbol{T}_{\boldsymbol{A}}$ equal to $0.75 T_{g l}$ over the time $\boldsymbol{t}_{\boldsymbol{A}}$, which far exceeds the crystallization time $\boldsymbol{t}_{\mathrm{Cr}}$ defined in advance in the process of studying the dynamics of crystallization.

\section{state}

\subsection{Stability of metal glasses in an amorphous}

Analyzing the results of the high-mentioned studies and the results of crystallization kinetics for metal amorphous stops of the metal-metal and metal - metalloid classes, the following is established. We have proposed in [3] such characteristic parameters as the temperature exposure $\boldsymbol{B}=\left(0.75 \boldsymbol{T}_{C r} \boldsymbol{t}_{A}\right)$, which determines the beginning of crystallization processes in the studied samples, and the crystallization time $\boldsymbol{t}_{C r}-$ are expedient to apply for estimating the probable time of their operation $\boldsymbol{t}_{\boldsymbol{O}}$ without risk of development of crystallization, dependent on the operating temperature. As a result, an empirical dependence of the link of required values is obtained, which agrees satisfactory with the experimental data:

$$
t_{O p}=A \sqrt{\frac{B_{t_{C r}}}{T_{O p}}},
$$

where $A$ is the factor equal to $600 \ldots 800$ that depends on the individual characteristics of the sample. Fig. 2 shows the dependence of $\boldsymbol{t}_{\boldsymbol{O}}$ for metal glasses of $\boldsymbol{T i}-\boldsymbol{C u}$ $\mathrm{Ni}$-Si class on the operating temperature $T_{O p}$.

\section{Conclusions}

The results of the research indicate that metallic glasses can be in an amorphous metastable state for a long time of operation. In particular, for example, the $\mathbf{T i}-\mathbf{C u}$ $\mathbf{N i}-\mathrm{Si}$ alloys at $373 \mathrm{~K}$ this time exceeds 50 years, and at $600 \mathrm{~K}-20$ years. Taking into account the exceptional stability of their electrical characteristics over time (change in the value of electrical resistance is $10^{-9}$ per year), we prove the effectiveness of their use in electro thermometry as materials for manufacturing the sensitive elements of the transducers.

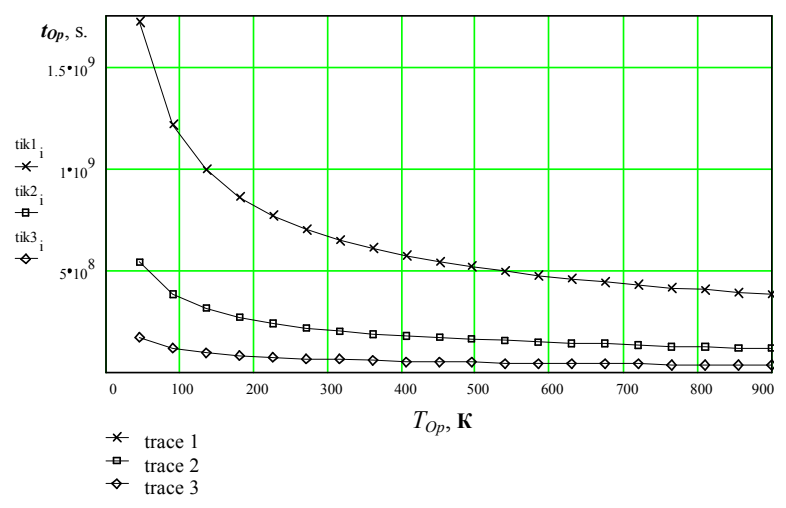

Fig. 2. Dependences of $\boldsymbol{t}_{O p}$ of $\mathbf{T i}-\mathbf{C u}$-Ni-Si metallic glasses on the operation temperature and the dynamics of the crystallization ( 1 for $\boldsymbol{t}_{C r}=1000 \mathrm{~s}$; 2 for $\boldsymbol{t}_{O p}=100 \mathrm{~s}$.; 3 for $\boldsymbol{t}_{O p}=10 \mathrm{~s}$.)

\section{Acknowledgments}

The authors express their gratitude to the staff of the Department of Information-Measuring Technologies of Lviv Polytechnic National University, Ukraine, for the assistance in the preparation of this article.

\section{Conflict of Interests}

Conflict of interest while writing, preparing, and publishing the article as well as mutual claims by the coauthors is absent.

\section{References}

[1] M. A. B. Mendesa, C. Sh. Kiminami, W. J. B. Filho, C. Bolfarini, M. F. de Oliveira, M. J. Kaufman, "Crystallization Behavior of Amorphous Ti51.1Cu38.9Ni10.0 Alloy”, Materials Research, vol. 18, pp. 104-108, 2015.

[2] R. Wang, "Phase Stability of Amorphous Intermetallic Alloys",Conference: AIME annual meeting, New Orleans, pp. 472-475, 1980.

[3] B. Stadnyk, P. Skoropad, S. Yatsyshyn, "Criteria for assessing the temperature stability of thermoelectrodes made of metal glasses", Journal of Thermoelectricity, no. 3, pp. 77-81, 2002.

[4] W. L. Johnson, "Fundamental Aspects of Bulk Metallic Glass Formation in Multicomponent Alloys", Materials Science Forum, vol. 225-227, pp. 35-50, 1996.

[5] I. H. Hillier, "Modified avrami equation for the bulk crystallization kinetics of spherulitic polymers", Journal of Polymer Science, vol. 3, no. 9, 1965. 